\title{
On the Domination Number of Cartesian Product of Two Directed Cycles
}

\author{
Zehui Shao, ${ }^{1,2}$ Enqiang Zhu, ${ }^{3}$ and Fangnian Lang ${ }^{1,2}$ \\ ${ }^{1}$ School of Information Science and Technology, Chengdu University, Chengdu 610106, China \\ ${ }^{2}$ Key Laboratory of Pattern Recognition and Intelligent Information Processing, Institutions of Higher Education of \\ Sichuan Province, Chengdu 610106, China \\ ${ }^{3}$ School of Electronic Engineering and Computer Science, Peking University, Beijing 100871, China
}

Correspondence should be addressed to Fangnian Lang; fnlang56@163.com

Received 18 September 2013; Revised 11 October 2013; Accepted 11 October 2013

Academic Editor: Carla Roque

Copyright (C) 2013 Zehui Shao et al. This is an open access article distributed under the Creative Commons Attribution License, which permits unrestricted use, distribution, and reproduction in any medium, provided the original work is properly cited.

Denote by $\gamma(G)$ the domination number of a digraph $G$ and $C_{m} \square C_{n}$ the Cartesian product of $C_{m}$ and $C_{n}$, the directed cycles of length $m, n \geq 2$. In 2010, Liu et al. determined the exact values of $\gamma\left(C_{m} \square C_{n}\right)$ for $m=2,3,4,5,6$. In 2013, Mollard determined the exact values of $\gamma\left(C_{m} \square C_{n}\right)$ for $m=3 k+2$. In this paper, we give lower and upper bounds of $\gamma\left(C_{m} \square C_{n}\right)$ with $m=3 k+1$ for different cases. In particular, $\lceil(2 k+1) n / 2\rceil \leq \gamma\left(C_{3 k+1} \square C_{n}\right) \leq\lfloor(2 k+1) n / 2\rfloor+k$. Based on the established result, the exact values of $\gamma\left(C_{m} \square C_{n}\right)$ are determined for $m=7$ and 10 by the combination of the dynamic algorithm, and an upper bound for $\gamma\left(C_{13} \square C_{n}\right)$ is provided.

\section{Introduction}

All of the digraphs considered in this paper are finite and simple, that is, without multiple edges or loops. For a digraph $G=(V, E)$ and a vertex $v \in V, N_{G}^{+}(v)$ and $N_{G}^{-}(v)$ denote the set of out-neighbors and in-neighbors of $v$. Given two vertices $u$ and $v$ in $G$, we say $u$ dominates $v$ if $u=v$ or $u v \in E$. Let $N_{G}^{+}[v]=N_{G}^{+}(v) \cup\{v\}$. A vertex $v$ dominates all vertices in $N_{G}^{+}[v]$. A set $D \subseteq V$ is a dominating set of $G$ if $D$ dominates $V(G)$. The domination number of $G$, denoted by $\gamma(G)$, is the minimum cardinality of a dominating set of $G$.

The Cartesian product of graphs $G$ and $H$ is the graph $G \square H$ with the vertex set $G \times H$, and $(g, h)\left(g^{\prime}, h^{\prime}\right) \in$ $E(G \square H)$ if either $g g^{\prime} \in E(G)$ and $h=h^{\prime}$ or $h h^{\prime} \epsilon$ $E(H)$ and $g=g^{\prime}$. The Cartesian product is commutative and associative, having the one-vertex graph as a unit. The subgraph of $G \square H$ induced by $V(G) \times h$, where $h \in V(H)$, is isomorphic to $G$, called a G-layer (over $h$ ) and denoted by $G^{h}$. For more information on the Cartesian product of graphs see [1].

We use a 0 - 1 matrix pattern $\mathscr{A}$ with $m$ rows and $n$ columns to represent a dominating set $D$ of $C_{m} \square C_{n}$, where $(i, j) \in D$ if and only if the value at the entry $(i, j)$ of $\mathscr{A}$ equals 1 . For example, the pattern in Figure 2 represents the dominating set (the set of black circles) shown in Figure 1. Let $P$ be a pattern; we denote by $c(P)$ the column number of $P$. Let $A$ and $B$ be patterns; then the pattern $A B$ denotes the concatenation of patterns $A$ and $B$, and $A^{k}$ denotes the concatenation of patterns $A$ for $k$ times.

Let $[n]$ denote the set $\{0,1, \ldots, n-1\}$. For an integer $n \geq 3$, the directed cycle of length $n$ is the graph $C_{n}$ whose vertices are $0,1, \ldots, n-1$ and whose edges are the pairs $i, i+1$, where the arithmetic is done modulo $n$. We denote $V\left(C_{n}\right)$ by $[n]$. In this paper, when we consider the Cartesian product graph $C_{m} \square C_{n}$ of two cycles $C_{m}$ and $C_{n}$, the edge set of $C_{m}$ is $\{(i, i+1): i \epsilon$ $[m]\}$ and $C_{n}$ is $\{(i+1, i): i \in[n]\}$. Throughout the paper, when considering the vertex $(i, j)$ in the graph $C_{m} \square C_{n}$, we use the arithmetic operations of the index $i$ over modulo $m$ and $j \operatorname{modulo} n$.

The dominating set problem requires determining the domination number of a given graph. It has natural applications in numerous facility location problems. In such problems, the vertices of a graph correspond to locations, adjacency represents some notion of accessability, and the goal is to find a subset of locations accessible from all other locations at which to install fire stations, bus stops, post 


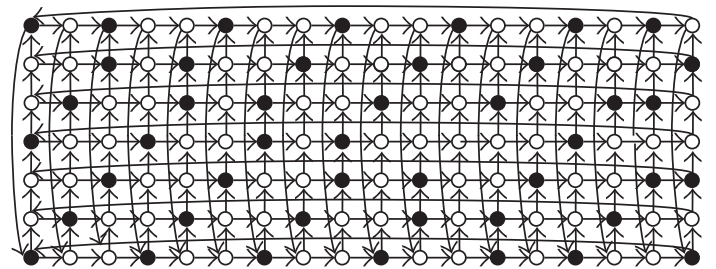

Figure 1: A dominating set of $C_{7} \square C_{18}$.

101001001001001010 001010010010010001 010010100100100110 100100101001001000 001001001010010011 010010010010100100 100100100100101001

Figure 2: Pattern of the dominating set shown in Figure 1.

offices, or similar facilities [2]. Dominating sets have also been applied in coding theory [2] and social networks [3]. For more information on the history and applications of the dominating set problem, see [4].

In 1990, Faudree and Schelp [5] initially discussed the domination number of the Cartesian product of two undirected graphs. The domination number for Cartesian product of two graphs has attracted lots of attention, and there are many works on undirected graphs (see, e.g., [6-12]). Recently, there are also some works on the domination number of Cartesian product of two directed graphs, in particular, the Cartesian product of directed paths and cycles [13-15].

In $[13,15]$, Liu et al. determined the exact values of $\gamma\left(C_{m} \square C_{n}\right)$ for $m=2,3,4,5,6$ and showed the following.

Theorem 1. $\gamma\left(C_{m} \square C_{n}\right)=m n / 3$ if $m \equiv 0(\bmod 3)$ and $n \equiv$ $0(\bmod 3)$.

Mollard [14] determined exact values of $\gamma\left(C_{m} \square C_{n}\right)$ for $m=3 k+2$. This paper investigates properties on the domination of Cartesian product of $C_{m}$ and $C_{n}$ with $m=$ $3 k+1$. We give lower and upper bounds of $\gamma\left(C_{m} \square C_{n}\right)$ with $m=3 k+1$ for different cases. In particular, $\lceil(2 k+1) n / 2\rceil \leq$ $\gamma\left(C_{3 k+1} \square C_{n}\right) \leq\lfloor(2 k+1) n / 2\rfloor+k$. Based on the established result, the exact values of $\gamma\left(C_{m} \square C_{n}\right)$ are determined for $m=7$ and 10 by the combination of the dynamic algorithm, and an upper bound for $\gamma\left(C_{13} \square C_{n}\right)$ is provided.

\section{Main Results}

2.1. Bounds on $C_{3 k+1} \square C_{n}$

Proposition 2 (Mollard [14]). Let $S$ be a dominating set of $C_{m} \square C_{n}$; then for all $i \in[n]$ considered modulon one has $\left|S \cap C_{m}^{i-1}\right|+2\left|S \cap C_{m}^{i}\right| \geq m$.

Lemma 3. Let $n \geq 3, k \geq 1$. Then there exists a minimum dominating set $D$ of $C_{3 k+1} \square C_{n}$ such that $\left|C_{3 k+1}^{i} \cap D\right| \geq k$ for every $i \in V\left(C_{n}\right)$.
Proof. For a dominating set $D$ of $C_{3 k+1} \square C_{n}$, we define a function $f$ as follows:

$$
f(D)=\sum_{t=0}^{n-1} x_{t}
$$

where

$$
x_{t}= \begin{cases}0, & \text { if }\left|D \cap C_{3 k+1}^{t}\right| \geq k+1, \\ \left|D \cap C_{3 k+1}^{t}\right|, & \text { if }\left|D \cap C_{3 k+1}^{t}\right| \leq k\end{cases}
$$

We now assume that $D$ is a minimum dominating set of $C_{3 k+1} \square C_{n}$ and suppose to the contrary that $\left|C_{3 k+1}^{i+1} \cap D\right|<k$ for some $i$ such that $f(D)$ is maximized. Then there exists a set $T=\{(j, i+1),(j+1, i+1),(j+2, i+1)\}$ such that $u \notin D$ for any $u \in T$. Thus, $\{(j, i),(j+1, i)\} \subseteq D$. By Proposition 2 , we have $x_{t}=0$ if $0<x_{t+1} \leq k$. Thus $D^{\prime}=D \backslash\{(j+1, i)\} \cup\{(j+1, i+1)\}$ is a dominating set with $|D|=\left|D^{\prime}\right|$ such that $f\left(D^{\prime}\right)>f(D)$, a contradiction with the fact that $f(D)$ is maximized.

Lemma 4. Let $n \geq 3, k \geq 1$. Then there exists a minimum dominating set $D$ of $C_{3 k+1} \square C_{n}$ such that $\left|\left(C_{3 k+1}^{i} \cup C_{3 k+1}^{i+1}\right) \cap D\right| \geq$ $2 k+1$ for every $i \in V\left(C_{n}\right)$.

Proof. By Lemma 3, it is sufficient to consider a minimum dominating set $D$ of $C_{3 k+1} \square C_{n}$ such that $\left|C_{3 k+1}^{i} \cap D\right| \geq k$ for every $i \in V\left(C_{n}\right)$. If $\left|C_{3 k+1}^{i} \cap D\right|=k$, together with Proposition 2, we have that $\left|C_{3 k+1}^{i-1} \cap D\right| \geq k+1$, which completes the proof.

By Lemmas 3 and 4, there is an obvious lower bound in terms of $\gamma\left(C_{3 k+1} \square C_{n}\right)$ as follows.

Lemma 5. Let $k \geq 1$ and $n \geq 3$ be two integers; then

$$
\gamma\left(C_{3 k+1} \square C_{n}\right) \geq\left\lceil\frac{(2 k+1) n}{2}\right\rceil .
$$

Lemma 6. For two integers $k \geq 1$ and $n \geq 3$, then for any $\ell \in[k]$, one has

(1) $\gamma\left(C_{3 k+1} \square C_{n}\right) \leq\lfloor(2 k+1) n / 2\rfloor+\ell$ if $n \equiv 6 \ell(\bmod 6 k+2)$,

(2) $\gamma\left(C_{3 k+1} \square C_{n}\right) \leq\lfloor(2 k+1) n / 2\rfloor+k-\ell$ if $n \equiv 2+$ $6 \ell(\bmod 6 k+2)$

(3) $\gamma\left(C_{3 k+1} \square C_{n}\right) \leq\lfloor(2 k+1) n / 2\rfloor+k$ if $n \equiv 4+6 \ell(\bmod 6 k+$ 2),

(4) $\gamma\left(C_{3 k+1} \square C_{n}\right) \leq\lfloor(2 k+1) n / 2\rfloor+\ell+1$ if $n \equiv 3+$ $6 \ell(\bmod 6 k+2)$,

(5) $\gamma\left(C_{3 k+1} \square C_{n}\right) \leq\lfloor(2 k+1) n / 2\rfloor+k-\ell$ if $n \equiv 5+$ $6 \ell(\bmod 6 k+2)$

(6) $\gamma\left(C_{3 k+1} \square C_{n}\right) \leq\lfloor(2 k+1) n / 2\rfloor+k$ if $n \equiv 7+6 \ell(\bmod 6 k+$ 2),

(7) $\gamma\left(C_{3 k+1} \square C_{n}\right) \leq\lfloor(2 k+1) n / 2\rfloor+k$ for $n \equiv 1(\bmod 6 k+2)$.

Proof. For any $i \in[n]$, let

$$
A_{i}= \begin{cases}\left\{\left(3 q+\frac{i}{2}, i\right): 0 \leq q \leq k\right\}, & \text { if } n \text { is even, } \\ \left\{\left(3 q+2+\left\lfloor\frac{i}{2}\right\rfloor, i\right): 0 \leq q \leq k-1\right\}, & \text { if } n \text { is odd. }\end{cases}
$$


It can be seen that if $i \equiv 0(\bmod 2),\left|A_{i}\right|=k+1$; otherwise, $\left|A_{i}\right|=k$. Let $S=\bigcup_{i=0}^{n-1} A_{i}$; then $|S|=\lceil(2 k+1) n / 2\rceil$ and there are at most $k$ vertices, $(1,0),(4,0),(7,0), \ldots,(3 k-2,0)$ of $C_{3 k+1} \square C_{n}$ of which each is not dominated by $S$. Denoted by $B=\{(1, n-1),(4, n-1), \ldots,(3 k-2, n-1)\}$, we consider the following cases.

(1) $n \equiv 6 \ell(\bmod 6 k+2)$. In this case, it is easy to see that $\left|A_{n-1} \cap B\right|=k-\ell$. By adding at most $\ell$ vertices to $S$, we get a dominating set of $\gamma\left(C_{3 k+1} \square C_{n}\right)$ as required.

(2) $n \equiv 2+6 \ell(\bmod 6 k+2)$. Since $\left|A_{n-1} \cap B\right|=\ell$, we will obtain a desired dominating set of $\gamma\left(C_{3 k+1} \square C_{n}\right)$ by adding at most $k-\ell$ vertices to $S$.

(3) $n \equiv 4+6 \ell(\bmod 6 k+2)$. Since $\left|A_{n-1} \cap B\right|=\varnothing$, we have to add at most $k$ vertices so that a dominating set of $\gamma\left(C_{3 k+1} \square C_{n}\right)$ is constructed based on $S$.

(4) $n \equiv 3+6 \ell(\bmod 6 k+2)$. Since $n-1$ is an even and $\mid A_{n-1} \cap$ $B \mid=k-\ell$, we have to add at most $\ell$ vertices to $S$ so that a dominating set of $\gamma\left(C_{3 k+1} \square C_{n}\right)$ will be formed.

(5) $n \equiv 5+6 \ell(\bmod 6 k+2)$. Since $\left|A_{n-1} \cap B\right|=\ell$, we will obtain a dominating set of $\gamma\left(C_{3 k+1} \square C_{n}\right)$ by adding at most $k-\ell$ vertices to $S$.

(6) $n \equiv 7+6 \ell(\bmod 6 k+2)$. Let $A_{n-3}^{\prime}=\{(1+3 \ell+3 k, n-$ $3),(2+3 \ell, n-3),(5+3 \ell, n-3), \ldots,(2+3 \ell+3(k-1), n-3)\}$ when $2+3 \ell>2+3 \ell+3 k(\bmod 3 k+1)$; otherwise, let $A_{n-3}^{\prime}=$ $\{(1+3 \ell, n-3),(5+3 \ell, n-3),(8+3 \ell, n-3), \ldots,(2+3 \ell+3 k, n-3)\}$ when $2+3 \ell<2+3 \ell+3 k(\bmod 3 k+1)$; let $A_{n-2}^{\prime}=C_{3 k+1}^{n-2} \backslash\{(1, n-$ $2),(4, n-2),(7, n-2), \ldots,(3 k-2, n-2),(1+3 \ell+3 k, n-2)\}$ when $2+3 \ell>2+3 \ell+3 k(\bmod 3 k+1)$; otherwise, let $A_{n-2}^{\prime}=C_{3 k+1}^{n-2} \backslash$ $\{(1, n-2),(4, n-2),(7, n-2), \ldots,(3 k-2, n-2),(1+3 \ell, n-2)\}$ when $2+3 \ell<2+3 \ell+3 k(\bmod 3 k+1)$; let $A_{n-1}^{\prime}=\{(1, n-$ $1),(4, n-1), \ldots,(3 k-2, n-1)\}, A_{i}^{\prime}=A_{i}$ for $i \in[n-4]$. In this way, if we let $D=\bigcup_{i=0}^{n-1} A_{i}^{\prime}$, then $D$ is a dominating set of $C_{3 k+1} \square C_{n}$. In addition, $\left|A_{n-3}^{\prime}\right|=k+1,\left|A_{n-2}^{\prime}\right|=2 k$, and $\left|A_{n-1}^{\prime}\right|=k$. So, $D=(((2 k+1) n) / 2)+((2 k-1) / 2)=$ $\lfloor((2 k+1) n) / 2\rfloor+k$.

(7) $n \equiv 1(\bmod 6 k+2)$. Let $D=\sum_{i=0}^{n-2} A_{i} \cup\{(1+3 q, n-1)$, (3+ $3 q, n-1): 0 \leq q \leq k-1\}$. Then $D$ is a dominating set of $C_{3 k+1} \square C_{n}$ with cardinality $\lfloor(2 k+1) n / 2\rfloor+k$.

The result of Lemmas 5 and 6 show the following.

Corollary 7. Let $k \geq 1$ and $n \geq 3$ be two integers; then

$$
\left\lceil\frac{(2 k+1) n}{2}\right\rceil \leq \gamma\left(C_{3 k+1} \square C_{n}\right) \leq\left\lfloor\frac{(2 k+1) n}{2}\right\rfloor+k .
$$

\section{On the Domination of $C_{7} \square C_{n}$}

Lemma 8. Let $k \geq 1$ and $n \geq 3$ be two integers. Suppose $D$ is a minimum dominating set of $C_{3 k+1} \square C_{n}$. Let $S_{i}=D \cap C_{3 k+1}^{i}$ and $a_{i}=\left|S_{i}\right|$ for $i=0,1, \ldots, n-1$.

(a) If there is an integer $i$ such that $a_{i}+a_{i+1}=2 k+1$ (considered modulon), then one of $\left\{a_{i}, a_{i+1}\right\}$ is $k$ and the other is $k+1$. (b) If there is an integer $i$ such that $a_{i}=k+1, a_{i+1}=k$, $a_{i+2}=k+1$, and $a_{i+3}=k$, then for any distinct vertices $\left(j_{1}, i+1\right),\left(j_{2}, i+1\right)$ in $S_{i+1}$ and $\left(j_{3}, i+3\right),\left(j_{4}, i+3\right)$ in $S_{i+3}$, $j_{1}-j_{2} \equiv 0(\bmod 3), j_{3}-j_{4} \equiv 0(\bmod 3) ;$ for any distinct vertex $\left(j_{1}^{\prime}, i\right),\left(j_{2}^{\prime}, i\right)$ in $S_{i}$ and $\left(j_{3}^{\prime}, i+2\right),\left(j_{4}^{\prime}, i+2\right)$ in $S_{i+2}$, $\left|j_{1}^{\prime}-j_{2}^{\prime}\right| \equiv 0(\bmod 3)$ or $\left|j_{1}^{\prime}-j_{2}^{\prime}\right|=1$ and $\left|j_{3}^{\prime}-j_{4}^{\prime}\right| \equiv$ $0(\bmod 3)$ or $\left|j_{3}^{\prime}-j_{4}^{\prime}\right|=1$, where $j_{1}<j_{2}, j_{3}<j_{4}$, $j_{1}^{\prime}<j_{2}^{\prime}, j_{3}^{\prime}<j_{4}^{\prime}$, and they are taken modulo $3 k+1$.

Proof. It follows immediately that (a) holds by Lemmas 3 and 4. In addition, since $a_{i}=k+1, a_{i+1}=k, a_{i+2}=k+1$, and $a_{i+3}=k$, each of vertices of $C_{3 k+1}^{i+1}$ and $C_{3 k+1}^{i+3}$ is dominated by only one vertex; also, only one vertex of $C_{3 k+1}^{i+2}$ is dominated by two vertices. Now we will first show that it is impossible that there are three consecutive vertices in $S_{i}$. Otherwise, suppose that $(s, i),(s+1, i)$, and $(s+2, i)$ (modulo $3 k+1)$ are three consecutive vertices. We can deduce that vertices $(s+1, i+2)$ and $(s+2, i+3)$ are in $S_{i+2}$ and $S_{i+3}$, respectively, so $(s+1, i+3)$ is dominated by both $(s+1, i+2)$ and $(s+2, i+3)$, a contradiction.

Suppose that there are $\left(j_{1}, i+1\right),\left(j_{2}, i+1\right)\left(\right.$ or $\left(j_{3}, i+3\right)$, $\left.\left(j_{4}, i+3\right)\right)$ in $S_{i+1}\left(\right.$ or $\left.S_{i+3}\right)$ such that $j_{1}-j_{2} \neq \equiv 0(\bmod 3)$ (or $\left.j_{3}-j_{4} \neq \equiv 0(\bmod 3)\right)$. Then it is easy to show that there exist three consecutive vertices $\in S_{i}$ or three consecutive vertices $\left(s_{1}, i+2\right),\left(s_{1}+1, i+2\right)$, and $\left(s_{1}+2, i+2\right) \in S_{i+2}$ (modulo $\left.3 k+1\right)$, thus $\left(s_{1}, i+1\right),\left(s_{1}+1, i+1\right)$, and $\left(s_{1}+2, i+1\right)$ (modulo $3 k+1$ ) can only be dominated by $S_{i}$, and $\left(s_{1}+4, i+1\right) \in S_{i+1}$ since it is impossible that there are four consecutive vertices in $S_{i+3}$ by $a_{i+3}=k$. So, it is an easy task to prove that either $\left(s_{1}+3, i+\right.$ $2)$ is not dominated by $D$ or $\left(s_{1}+4, i+2\right)$ is dominated by two vertices, a contradiction. By using the same approach as above, we also can prove the cases of vertices in $S_{i}$ and $S_{i+2}$.

Lemma 9. Let $k \geq 1$ and $n \geq 3$. If $D$ is a minimum dominating set of $C_{3 k+1} \square C_{n}$ such that $\left(a_{i}, a_{i+1}, a_{i+2}, a_{i+3}, \ldots, a_{i+2 \ell}\right)=(k+$ $1, k, k+1, k, \ldots, k+1)$, where $S_{i}=D \cap C_{3 k+1}^{i}$ and $a_{i}=\left|S_{i}\right|$ for some $i, \ell$, then $(j, i+1) \in S_{i+1}$ if and only if $(j+t-1, i+2 t-1) \in$ $S_{i+2 t-1}$ for any $j \in\{0,1, \ldots, 3 k\}$ and $t \in\{1,2, \ldots, \ell\}$.

Proof. By Lemma 8 and the symmetrical structure of $C_{m} \square C_{n}$, we assume without loss of generality $S_{i+2 \ell-1}=\{(3 q, i+2 \ell-1)$ : $0 \leq q \leq k-1\}$. Since each vertex in $\{(3 q-1, i+2 \ell-1): 0 \leq$ $q \leq k-1\} \cup(-2, i+2 \ell-1)$ is not dominated by $C_{3 k+1}^{i+2 \ell-1}$, we have $S_{i+2 \ell-2}=\{(3 q-1, i+2 \ell-2): 0 \leq q \leq k-1\} \cup(-2, i+2 \ell-2)$. By observing that each vertex in $S_{i+2 \ell-2}$ needs to be dominated, we have the desired set $S_{i+2 \ell-3}$. Repeating this process until $S_{i+1}$ is determined, we have the desired result.

Theorem 10. Let $n \geq 7$. Then

$$
\begin{aligned}
& \gamma\left(C_{7} \square C_{n}\right) \\
& = \begin{cases}\left\lfloor\frac{5 n}{2}\right\rfloor, & n \equiv 0(\bmod 14), \\
\left\lfloor\frac{5 n}{2}\right\rfloor+1, & n \equiv 3,4,6,8,10,11(\bmod 14), \\
\left\lfloor\frac{5 n}{2}\right\rfloor+2, & n \equiv 1,2,5,7,9,12,13(\bmod 14) .\end{cases}
\end{aligned}
$$


101001010010100100100100

001010100100101001001001

010010001001001010010010

100100110010010010100100

001001000100100100101001

010010011001001001001010

100100101010010010010010

FIgURE 3: A dominating set of $C_{7} \square C_{24}$.

Proof. Consider the following.

Case $1 . n \equiv 0(\bmod 14)$.

The lower bound follows from Lemma 5, and the upper bound follows from Lemma 6 with $\ell=0$ in Case 1 .

Case 2. $n \equiv 3,4,6,8,10,11(\bmod 14)$.

By using Lemmas 3 and 4 , we obtain that $\gamma\left(C_{7} \square C_{n}\right)=$ $\lfloor 5 n / 2\rfloor$ if and only if $n \equiv 0(\bmod 14)$. Therefore, the desired lower bound is established. Upper bound can be obtained by applying Lemma 6 with $k=2$ in the following cases:

(i) $n \equiv 3(\bmod 14), \ell=0$ in case 4 ;

(ii) $n \equiv 6(\bmod 14), \ell=1$ in case 1 ;

(iii) $n \equiv 8(\bmod 14), \ell=1$ in case 2 ;

(iv) $n \equiv 11(\bmod 14), \ell=1$ in case 5 .

We give the upper bound for the case $n \equiv 4,10(\bmod 14)$ by constructions.

Let $n=14 k+4$. The pattern depicted in Figure 2 is a dominating set of $C_{7} \square C_{18}$ with 46 vertices. Moreover, the leftmost 14 columns induce a dominating set of $C_{7} \square C_{14}$ with 35 vertices. By repeating the leftmost 14 columns for $k$ times, we get a dominating set of $C_{7} \square C_{14 k+4}$ with $35 k+11$ vertices. Therefore, $\gamma\left(C_{7} \square C_{14 k+4}\right) \leq 35 k+11$, and thus, $\gamma\left(C_{7} \square C_{n}\right) \leq$ $\lfloor 5 n / 2\rfloor+1$.

Let $n=14 k+10$. The pattern depicted in Figure 3 is a dominating set of $C_{7} \square C_{24}$ with 61 vertices. Moreover, the leftmost 14 columns induce a dominating set of $C_{7} \square C_{14}$ with 35 vertices. By repeating the leftmost 14 columns for $k$ times, we get a dominating set of $C_{7} \square C_{14 k+10}$ with $35 k+26$ vertices. Therefore, $\gamma\left(C_{7} \square C_{14 k+10}\right) \leq 35 k+26$, and thus, $\gamma\left(C_{7} \square C_{n}\right) \leq$ $\lfloor 5 n / 2\rfloor+1$.

Case 3. $n \equiv 1,2,5,7,9,12,13(\bmod 14)$.

The upper bound follows from Lemma 6 . We then show the lower bound. Let $n \equiv 1(\bmod 14)$. If there exists a minimum dominating set of $C_{3 k+1} \square C_{n}$ with cardinality $\lfloor 5 n / 2\rfloor+1$, then by Lemmas 3 and 4 , we have such a dominating set $D$ with $\left(a_{0}, a_{1}, a_{2}, a_{3}, \ldots, a_{n-2}, a_{n-1}\right)=(3,2,3,2, \ldots, 2,3)$, where $S_{i}=D \cap C_{7}^{i}$ and $a_{i}=\left|S_{i}\right|$. We without loss of generality assume $S_{n-2}=\{(0, n-2),(3, n-2)\}$. Then $S_{1}=\{(1,1),(4,1)\}$ and so $S_{0}=\{(0,0),(3,0),(6,0)\}$. We now consider $S_{n-2}, S_{n-1}$, and $S_{0}$ with $S_{n-2}$ and $S_{0}$ known. Then there are four vertices in $S_{n-1}$ not dominated by $S_{n-2} \cup S_{0}$, a contradiction. Therefore, $\gamma\left(C_{7} \square C_{n}\right) \geq\lfloor 5 n / 2\rfloor+2$ if $n \equiv 1(\bmod 14)$. By using the same approach, we can obtain the desired lower bound if $n \equiv 2,5,7,9,12,13(\bmod 14)$ and the proof is omitted.

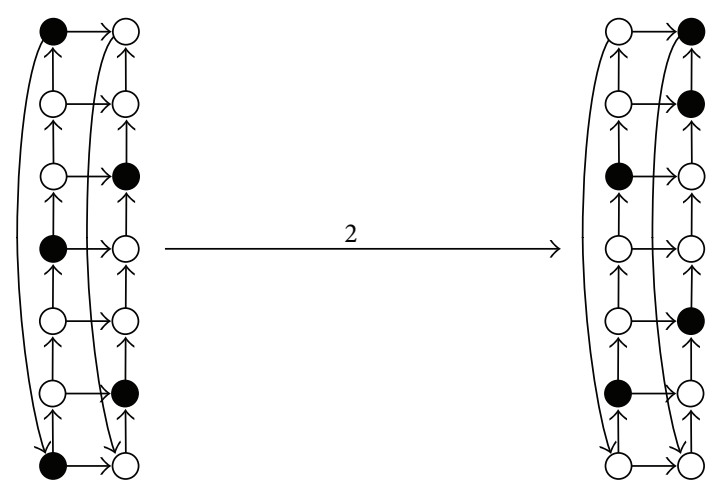

Figure 4: Two vertices of $D_{7}$.

\section{On the Domination of $C_{10} \square C_{n}$}

We use the dynamic algorithm to provide the result on $\gamma\left(C_{m} \square C_{n}\right)$ for $m=10$ and 13 . We first describe the concept needed to describe our computer checking. The idea is introduced in [16] in a more general framework and extended in [17], but for our purpose the following description will be sufficient.

For a graph $G$ and $D \subseteq V(G)$, we define a function $f$ : $V(G) \rightarrow\{0,1\}$ such that $f(v)=0$ if $v \notin D$ and $f(v)=1$ if $v \in D$. We call such a function the 2-coloring of $G$. If for $f(v)=0$ there exists a vertex $u \in N_{G}^{-}(v)$ such that $f(v)=1$, then we say $v$ is dominated. We can see that this notation is equivalent to the dominating set and we will use it to describe the dynamic algorithm for this problem.

Let $C=\left\{u_{i} u_{i+1}: i \in[n]\right\}$ be a directed cycle in a weighted digraph $G$. We say that the sum of the weights of all the edges in $C$ to be the weight of $C$.

Let $u$ be a 2-coloring of $C_{m} \square P_{2} ; u$ is said to be an $(a, b)$ vertex if the number of ones in the first copy of $C_{m}$ equals $a$ and the second equals $b$ (see, e.g., the left side of the vertex in Figure 4 is a $(3,2)$-vertex). An $(a, b)$-vertex is said to be redundant if it is dominated and any removal of one vertex in the first column destroys the domination.

Let $T=\left\{\left(a_{i}, b_{i}\right): 1 \leq i \leq t\right\}$. We define a weighted digraph $D_{m}^{T}$ (when no confusion can arise, the set $T$ is omitted and the digraph is denoted by $D_{m}$ ) as follows.

The vertices of $D_{m}^{T}$ consist of all the nonredundant 2colorings of $C_{m} \square P_{2}$. Let $u=u_{1} u_{2}$ be a vertex of $D_{m}^{T}$. Then $u_{1}$ and $u_{2}$ represent the 2 -colorings of $C_{m} \square P_{2}$ restricted to the first and second copies of $C_{m}$. Let $u$ and $v$ be two vertices of $D_{m}^{T}$. Then $\widetilde{u v}$ denotes the 2-coloring of $C_{m} \square P_{3}$ obtained by applying $u_{2}$ and $v_{1}$ to the consecutive copies of $C_{m}$. For each vertex $u$ of $D_{m}^{T}$, we make an arc from $u$ to $v$ in $D_{m}^{T}$ if and only if the following conditions are fulfilled:

(i) $u_{2}$ equals $v_{1}$,

(ii) each of the vertices of $C_{m}^{1} \cup C_{m}^{2}$ is dominated (by $C_{m}^{0} \cup$ $\left.C_{m}^{1}\right)$

(iii) for each $(a, b)$-vertex of $D_{m}^{T},(a, b) \in T$.

Finally, the weight of $u v$ is the number of ones in $C_{m}^{1}$ of $v$. 
Let $T=\{(2,3),(3,2)\}$. Figure 4 shows two vertices of $D_{7}^{T}$. We can see that the 2-coloring (0010010) of the second copy of $C_{7}$ of the first vertex equals the first copy of $C_{7}$ of the second vertex. Moreover, the weight of the second copy of $C_{7}$ of the first vertex is 2. It follows that $A\left(D_{7}^{T}\right)$ has an arc from the first (left) vertex to the second (right) with weight 2.

Lemma 11. Let $m, n \geq 3$. Then $D_{m}$ possesses a directed cycle of length $n$ with weight $k$ if and only if $C_{m} \square C_{n}$ contains a minimal dominating set $S$ with $|S|=k$.

Proof. If $S$ is a minimal dominating set of $C_{m} \square C_{n}$, then $S$ restricted to $C_{m}^{i} \cup C_{m}^{i+1}$ is a 2 -coloring $\mathscr{C}$ of $C_{m} \square P_{2}$. Since $S$ is minimal, we have that $\mathscr{C}$ is nonredundant.

If $D_{m}$ possesses a directed cycle of length $n$, we concatenate the second cycle $C_{m}$ of each vertex of the cycle and then obtain a dominating set $S$ of $C_{m} \square C_{n}$. Since each vertex in the cycle is nonredundant, we have that $S$ is minimal.

We define a weighted adjacency matrix $A\left(D_{m}\right)$ of $D_{m}$ as follows. Let $V\left(D_{m}\right)=\left\{v_{1}, v_{2}, \ldots, v_{K}\right\}$. Then

$$
A\left(D_{m}\right)_{i, j}= \begin{cases}w\left(v_{i} v_{j}\right), & v_{i} v_{j} \in D_{m}, \\ +\infty, & v_{i} v_{j} \notin D_{m},\end{cases}
$$

where $w\left(v_{i} v_{j}\right)$ is the weight of the edge $v_{i} v_{j}$.

Let $A=(a)_{p \times q}$ ( $p$ rows, $q$ columns) and $B=(b)_{q \times r}(q$ rows, $r$ columns) be two weighted adjacency matrices. We define $A$ multiplied by $B$ as follows

$$
\begin{gathered}
\left(\begin{array}{cccc}
a_{1,1} & a_{1,2} & \cdots & a_{1, q} \\
a_{2,1} & a_{2,2} & \cdots & a_{2, p} \\
\vdots & \vdots & \vdots & \vdots \\
a_{p, 1} & a_{p, 2} & \cdots & a_{p, q}
\end{array}\right) \times\left(\begin{array}{cccc}
b_{1,1} & b_{1,2} & \cdots & b_{1, r} \\
b_{2,1} & b_{2,2} & \cdots & b_{2, r} \\
\vdots & \vdots & \vdots & \vdots \\
b_{q, 1} & b_{q, 2} & \cdots & b_{q, r}
\end{array}\right) \\
=\left(\begin{array}{cccc}
c_{1,1} & c_{1,2} & \cdots & c_{1, r} \\
c_{2,1} & c_{2,2} & \cdots & c_{2, r} \\
\vdots & \vdots & \vdots & \vdots \\
c_{p, 1} & c_{p, 2} & \cdots & c_{p, r}
\end{array}\right),
\end{gathered}
$$

where $c_{s, t}=\min _{1 \leq i \leq q}\left\{a_{s, i}+b_{i, t}\right\}$ for $s \in\{1,2, \ldots, p\}$ and $t \in$ $\{1,2, \ldots, r\}$.

We denote by $A^{n}$ the $n$th power of $A$; we have the following result.

Theorem 12. Let $m, n \geq 3$ and $A\left(D_{m}\right)$ be the weighted adjacency matrix of $D_{m}$ with $K$ vertices. Then one has

$$
\gamma\left(C_{m} \square C_{n}\right)=\min \left\{\left(\left(A\left(D_{m}\right)\right)^{n}\right)_{i, i}: 1 \leq i \leq K\right\} .
$$

Lemma 13. Let $n \geq 10, \gamma\left(C_{10} \square C_{n}\right) \leq\lfloor 7 n / 2\rfloor+2$, and $D a$ dominating set with cardinality $\lfloor 7 n / 2\rfloor+2$.

(a) If $n$ is odd, then $\left|D \cap\left(C_{10}^{i} \cup C_{10}^{i+1}\right)\right| \leq 8$ for any $i \in V\left(C_{n}\right)$.

(b) If $n$ is even, then $\left|D \cap\left(C_{10}^{i} \cup C_{10}^{i+1}\right)\right| \leq 9$ for any $i \in V\left(C_{n}\right)$ but at most one integer $i_{0}$ such that $\left|D \cap\left(C_{10}^{i_{0}} \cup C_{10}^{i_{0}+1}\right)\right|=$ 9.
Proof. (a) Suppose that there exists $i_{0} \in[n-1]$ such that $\mid D \cap$ $\left(C_{10}^{i_{0}} \cup C_{10}^{i_{0}+1}\right) \mid \geq 9$. We can deduce that

$$
\left|D \cap \bigcup_{\substack{j \in[n-1], j \notin\left\{i_{0}, i_{0}+1\right\}}} V\left(C_{10}^{j}\right)\right| \leq \frac{7}{2}(n-2)-\frac{1}{2} .
$$

Thus, by the pigeonhole principle it can be seen that there exist some $i \in[n-1]$ such that $\left|D \cap\left(C_{10}^{i} \cup C_{10}^{i+1}\right)\right|<7$, which contradicts with Lemma 4.

(b) The proof is similar to (a).

Theorem 14. Let $n \geq 10$. Then

$$
\begin{aligned}
& \gamma\left(C_{10} \square C_{n}\right) \\
& = \begin{cases}\left\lfloor\frac{7 n}{2}\right\rfloor, & n \equiv 0(\bmod 20), \\
\left\lfloor\frac{7 n}{2}\right\rfloor+1, & n \equiv 3,6,14,17(\bmod 20), \\
\left\lfloor\frac{7 n}{2}\right\rfloor+2, & n \equiv 4,7,8,9,10,11,12,13,16(\bmod 20), \\
\left\lfloor\frac{7 n}{2}\right\rfloor+3, & n \equiv 1,2,5,15,18,19(\bmod 20) .\end{cases}
\end{aligned}
$$

Proof. When $n \equiv 0,3,6,14,17(\bmod 20)$, the result follows from Lemmas 6, 8, and 9. Otherwise, let $T=$ $\{(3,4),(4,3),(3,5),(5,3),(4,4)\}$. We implemented the algorithm as described above, and the digraph $D_{10}^{T}$ was created and it has 4905 vertices. By Lemma 13, it can be seen that there may exist at most one $(x, y)$-vertex such that $x+y=9$ in the auxiliary graph $D_{10}$. We consider each possible auxiliary graph $D_{10}$, whose vertex set is the union of that of $D_{10}^{T}$ and one $(x, y)$-vertex with $x+y=9$. Note that $D_{10}$ has only 4906 vertices; the matrix multiplication can be done easily. By using matrix multiplication, we found a formula as stated in Theorem 14. (Such parameters $a$ and $b$ exist; see [17].) If $\gamma\left(C_{10} \square C_{n}\right)$ (for some $n$ ) is greater than $\lfloor 7 n / 2\rfloor+2$, then $\gamma\left(C_{10} \square C_{n}\right)=\lfloor 7 n / 2\rfloor+3$.

Remark 15. Note that when Lemmas 4 and 13 are applied, we can operate matrix multiplication on the graph $D_{10}^{T_{1}}$ whose row number is 4906 . It can be clearly seen that $\mid D \cap\left(C_{10}^{i} \cup\right.$ $\left.C_{10}^{i+1}\right) \mid \leq 9$ for any $i \in V\left(C_{n}\right)$ with the condition of Lemma 13 , and it is natural to consider the graph $D_{10}^{T_{2}}$, where $T_{2}=T \cup\{(3,6),(4,5),(5,4),(6,3)\}$. However, $D_{10}^{T_{2}}$ has 24845 vertices, whose vertex number is much more than that of $D_{10}$. Therefore, Lemmas 4 and 13 play the key role in computing the exact values of $\gamma\left(C_{10} \square C_{n}\right)$, and this helps to make many hard instances become easy to solve. 
TABLE 1: Constructions for upper bounds of $C_{13} \square C_{n}$.

\begin{tabular}{lccccccccc}
\hline$r$ & 0 & 1 & 2 & 3 & 4 & 5 & 6 & 7 & 8 \\
$(c, \ell)$ & $(1,0)$ & $(7,0)$ & $(2,0)$ & $(4,0)$ & $A^{k} B$ & $(5,0)$ & $(1,1)$ & $A^{k} C$ & $(2,1)$ \\
\hline$r$ & 9 & 10 & 11 & 12 & 13 & 14 & 15 & 16 & 17 \\
$(c, \ell)$ & $(4,1)$ & $A^{k} D$ & $(5,1)$ & $(1,2)$ & $(6,1)$ & $(2,2)$ & $(4,2)$ & $A^{k} E$ & $(5,2)$ \\
\hline$r$ & 18 & 19 & 20 & 21 & 22 & 23 & 24 & 25 & $(1,4)$ \\
$(c, \ell)$ & $(1,3)$ & $A^{k} F$ & $(2,3)$ & $(4,3)$ & $A^{k} G$ & $(5,3)$ & $(6,3)$ & \\
\hline
\end{tabular}

\section{Bounds on the Domination Number of $C_{13} \square C_{n}$}

We will conclude this work with upper bounds for the domination number of $C_{13} \square C_{n}$.

Theorem 16. Let $n \geq 13$. Then

$$
\begin{aligned}
& \gamma\left(C_{13} \square C_{n}\right) \\
& \leq \begin{cases}\left\lfloor\frac{9 n}{2}\right\rfloor, & n \equiv 0(\bmod 26), \\
\left\lfloor\frac{9 n}{2}\right\rfloor+1, & n \equiv 3,6,20,23(\bmod 26), \\
\left\lfloor\frac{9 n}{2}\right\rfloor+2, & n \equiv 9,10,12,14,16,17(\bmod 26), \\
\left\lfloor\frac{9 n}{2}\right\rfloor+3, & n \equiv 4,7,8,11,13,15,18,19,22(\bmod 26), \\
\left\lfloor\frac{9 n}{2}\right\rfloor+4, & n \equiv 1,2,5,21,24,25(\bmod 26) .\end{cases}
\end{aligned}
$$

Proof. We show the desired weight for all cases in Table 1, where $r \equiv n(\bmod 26)$ and $(c, \ell)$ stands for case $(c)$ with $\ell$ in Lemma 6 . For example, the result of the case $n \equiv 0(\bmod 26)$ follows from case 1 with $\ell=0$ in Lemma 6 .

Also, patterns $A-G$ with 13 rows are presented (see Figures 5 and 6 ). By repeating the pattern of $A$ for $k$ times and concatenating $H \in\{B, C, D, E, F, G\}$, we will obtain a pattern $A^{k} H$ of $C_{13} \square C_{n}$, where $n=26 k+c(H)$. From the above, it can be seen that all the desired upper bounds are established.

\section{Disclosure}

The authors confirm that they have given due consideration to the protection of intellectual property associated with this work and that there are no impediments to publication, including the timing of publication, with respect to intellectual property. In so doing, the authors confirm that they have followed the regulations of their institutions concerning intellectual property. The authors understand that the corresponding author is the sole contact for the editorial process (including editorial manager and direct communications with the office). $\mathrm{He} /$ she is responsible for communicating with the other authors about progress, submissions of revisions, and final approval of proofs. The authors confirm that they have provided current, correct

$\begin{array}{cccc}01001001001010010010010010 & 0010 & 0100010 & 0100100010 \\ 10010010010010100100100100 & 1100 & 1001100 & 1001001100 \\ 00100100100100101001001001 & 0001 & 0010001 & 0010010001 \\ 01001001001001001010010010 & 0110 & 0100110 & 0100100110 \\ 10010010010010010010100100 & 1000 & 1001000 & 1001001000 \\ 00100100100100100100101001 & 0011 & 0010011 & 0010010011 \\ 01001001001001001001001010 & 0100 & 0100100 & 0100100100 \\ 10010010010010010010010010 & 1001 & 1001001 & 1001001001 \\ 10100100100100100100100100 & 1010 & 1010010 & 1010010010 \\ 00101001001001001001001001 & 1001 & 0000101 & 0010100101 \\ 01001010010010010010010010 & 0010 & 0111010 & 0100101010 \\ 10010010100100100100100100 & 1100 & 1000100 & 1001000100 \\ 00100100101001001001001001 & 1001 & 0011001 & 0010011001 \\ A & & & \end{array}$

Figure 5: $A B$ : dominating set of $C_{13} \square C_{30}$; $A C$ : dominating set of $C_{13} \square C_{33} ; A D$ : dominating set of $C_{13} \square C_{36}$. $\begin{array}{ll}0100100010010010 & 0100100010010010010 \\ 1001001100100100 & 1001001100100100100 \\ 0010010001001001 & 0010010001001001001 \\ 0100100110010010 & 0100100110010010010 \\ 1001001010100100 & 1001001010100100100 \\ 0010010100101001 & 0010010100101001001 \\ 0100101001001010 & 0100101001001010010 \\ 1001010010010010 & 1001010010010010101 \\ 1010100100100100 & 1010100100100100100 \\ 0010001001001001 & 0010001001001001001 \\ 0100110010010010 & 0100110010010010010 \\ 1001000100100100 & 1001000100100100100 \\ 0010011001001001 & 0010011001001001001\end{array}$

E $\quad F$
0100100100010010010010 0010010010101001001001 0100100101001010010010 1001001010010010100100 0010010100100100101001 0100101001001001001010 1001010010010010010010 1011010100100100100100 0010010001001001001001 1001001000100100100100 0010010011001001001001

G 1001001001100100100100 0100100110010010010010

FIgURE 6: $A E$ : dominating set of $C_{13} \square C_{42} ; A F$ : dominating set of $C_{13} \square C_{45}$; AG: dominating set of $C_{13} \square C_{48}$.

email addresses (fnlang56@163.com and zshao@cdu.edu.cn) which is accessible by the corresponding author and which has been configured to accept email from.

\section{Conflict of Interests}

The authors wish to confirm that there is no known conflict of interests associated with this paper and there has been no significant financial support for this work that could have influenced its outcome. The authors confirm that the paper has been read and approved by all named authors and that there are no other persons who satisfied the criteria for authorship but are not listed. The authors further confirm that the order of authors listed in the paper has been approved by all of them. 


\section{Acknowledgments}

The authors would like to acknowledge the referees for their valuable comments and suggestions. This work was supported by the National Natural Science Foundation of China under Grants nos. 61309015 and 61173121.

\section{References}

[1] R. Hammack, W. Imrich, and S. Klavžar, Handbook of Product Graphs, Discrete Mathematics and Its Applications, CRC Press, Boca Raton, Fla, USA, 2nd edition, 2011.

[2] I. Gorodezky, Domination in kneser graphs [Ph.D. thesis], University of Waterloo, Ontario, Canada, 2007.

[3] L. L. Kelleher and M. B. Cozzens, "Dominating sets in social network graphs," Mathematical Social Sciences, vol. 16, no. 3, pp. 267-279, 1988.

[4] T. W. Haynes, S. T. Hedetniemi, and P. J. Slater, Fundamentals of Domination in Graphs, vol. 208 of Monographs and Textbooks in Pure and Applied Mathematics, Marcel Dekker, New York, NY, USA, 1998.

[5] R. J. Faudree and R. H. Schelp, "The domination number for the product of graphs," Congressus Numerantium, vol. 79, pp. 29-33, 1990.

[6] M. El-Zahar, S. Khamis, and Kh. Nazzal, "On the domination number of the cartesian product of the cycle of length $n$ and any graph," Discrete Applied Mathematics, vol. 155, no. 4, pp. 515522, 2007.

[7] M. El-Zahar and C. M. Pareek, "Domination number of products of graphs," Ars Combinatoria, vol. 31, pp. 223-227, 1991.

[8] T. Y. Chang and W. E. Clark, "The domination numbers of the $5 \times n$ and $6 \times n$ grid graphs," Journal of Graph Theory, vol. 17, no. 1, pp. 81-107, 1993.

[9] S. Gravier and M. Mollard, "On domination numbers of cartesian product of paths," Discrete Applied Mathematics, vol. 80, no. 2-3, pp. 247-250, 1997.

[10] B. L. Hartnell and D. F. Rall, "On dominating the cartesian product of a graph and $\mathrm{K}_{2}$," Discussiones Mathematicae. Graph Theory, vol. 24, no. 3, pp. 389-402, 2004.

[11] M. S. Jacobson and L. F. Kinch, "On the domination number of products of graphs. I," Ars Combinatoria, vol. 18, pp. 33-44, 1984.

[12] S. Klavžar and N. Seifter, "Dominating cartesian products of cycles," Discrete Applied Mathematics, vol. 59, no. 2, pp. 129-136, 1995.

[13] J. Liu, X. Zhang, X. Chen, and J. Meng, "On domination number of cartesian product of directed cycles," Information Processing Letters, vol. 110, no. 5, pp. 171-173, 2010.

[14] M. Mollard, "On the domination of cartesian product of directed cycles: results for certain equivalence classes of lengths," Discussiones Mathematicae. Graph Theory, vol. 33, no. 2, pp. 387-394, 2013.

[15] X. Zhang, J. Liu, X. Chen, and J. Meng, "Domination number of cartesian products of directed cycles," Information Processing Letters, vol. 111, no. 1, pp. 36-39, 2010.

[16] S. Klavžar and J. Žerovnik, "Algebraic approach to fasciagraphs and rotagraphs," Discrete Applied Mathematics, vol. 68, no. 1-2, pp. 93-100, 1996.

[17] P. Pavlič and J. Žerovnik, "Roman domination number of the cartesian products of paths and cycles," Electronic Journal of Combinatorics, vol. 19, no. 3, p. 19, 2012. 


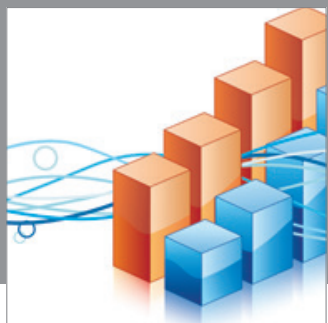

Advances in

Operations Research

mansans

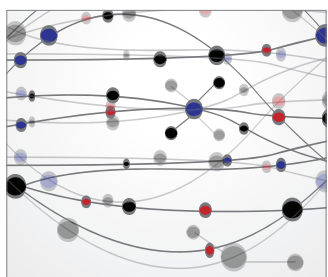

The Scientific World Journal
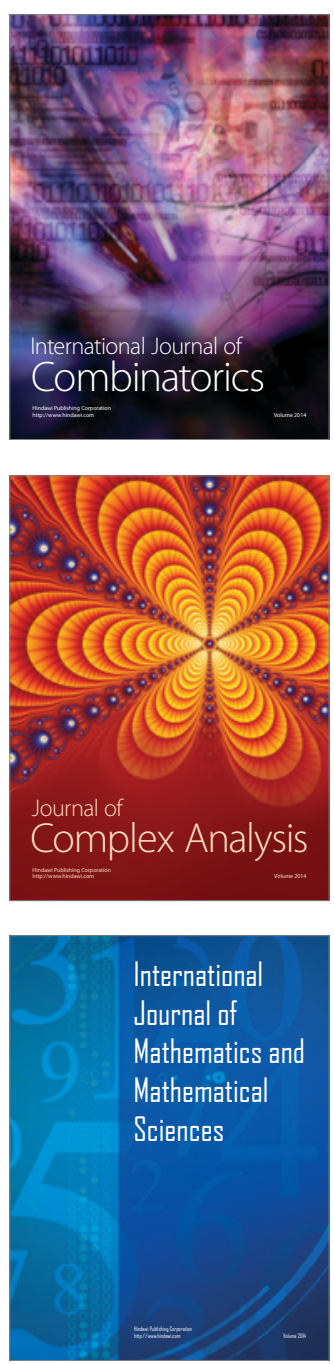
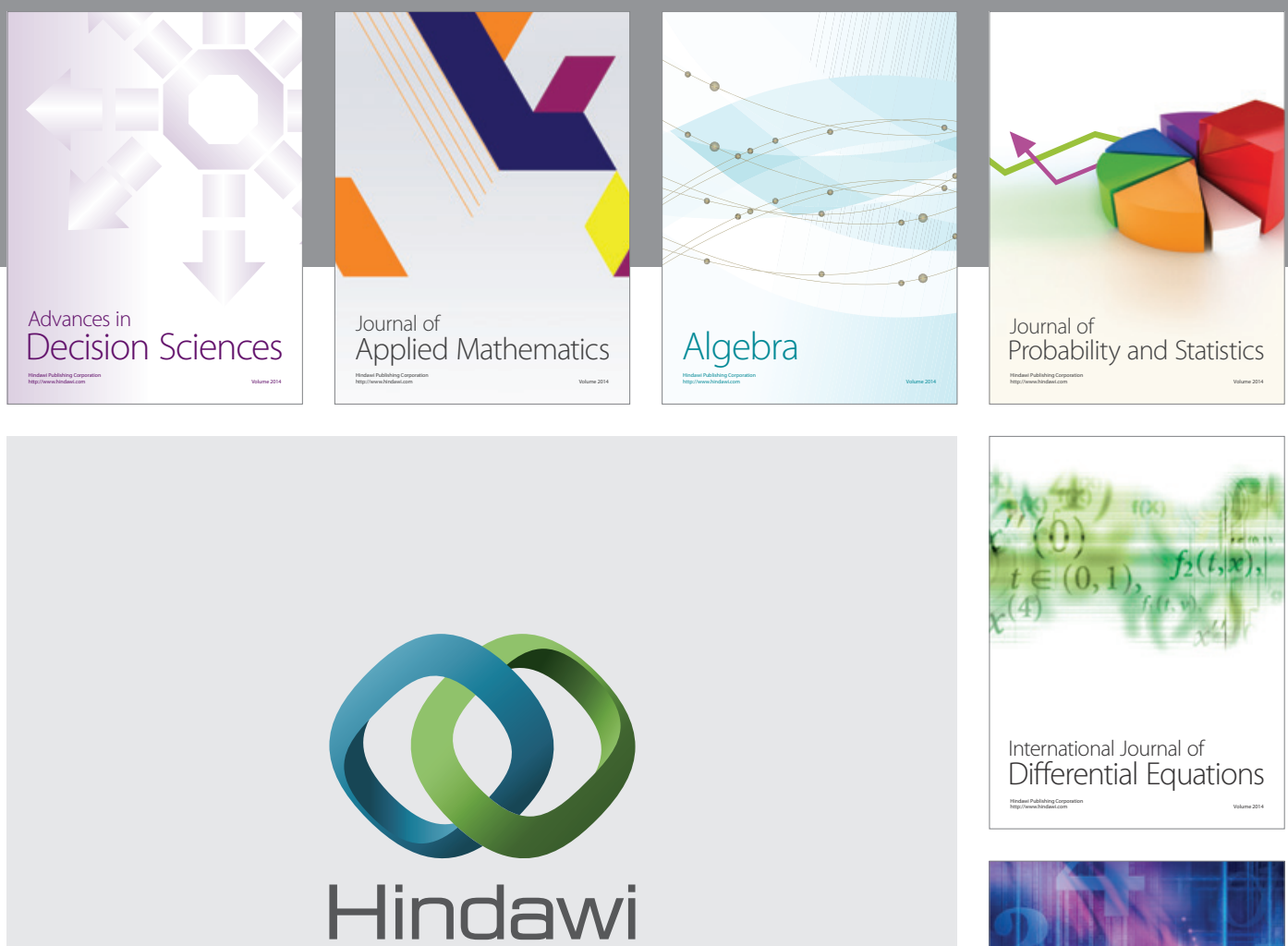

Submit your manuscripts at http://www.hindawi.com
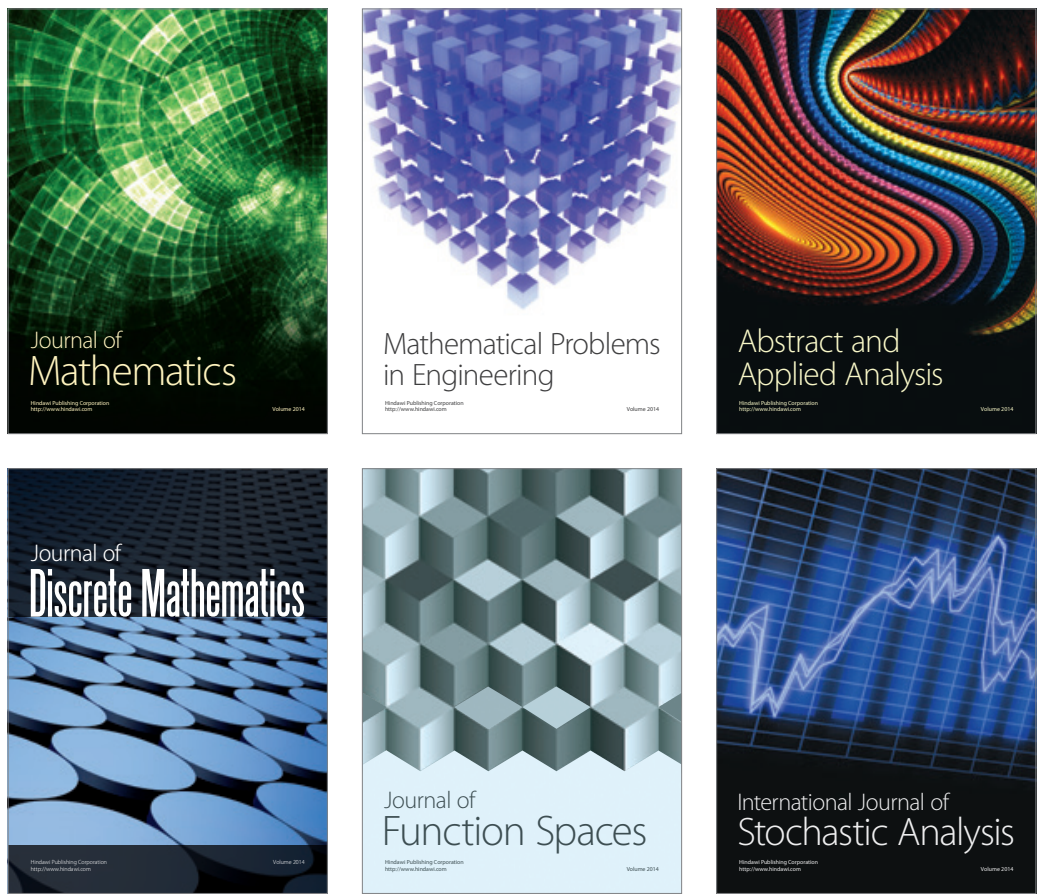

Journal of

Function Spaces

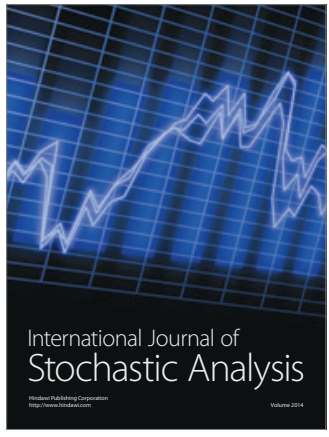

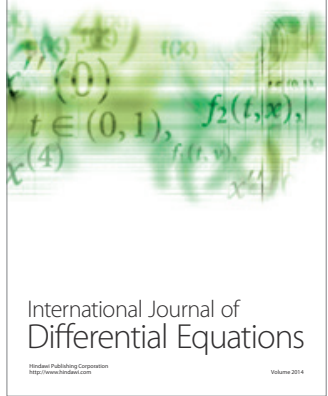
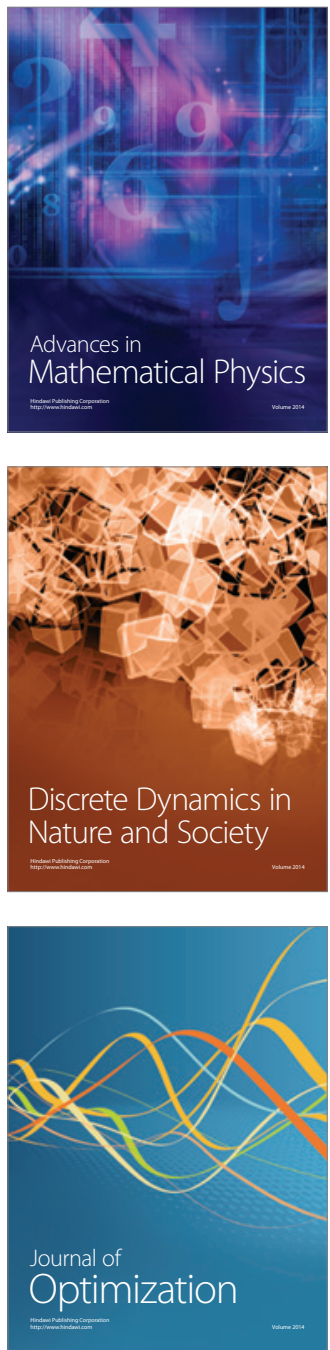